\title{
Russian geomagnetic recordings in 1850-1862 compared to modern observations
}

\author{
Ari Viljanen ${ }^{1, *}$, Minna Myllys ${ }^{1,2}$, and Heikki Nevanlinna ${ }^{1}$ \\ 1 Finnish Meteorological Institute, Erik Palmenin aukio 1, FI-00560 Helsinki, Finland \\ Corresponding author: ari.viljanen@fmi.fi \\ 2 Department of Physics, University of Helsinki, P.O. Box 64, FI-00014, Finland
}

Received 5 December 2013 / Accepted 3 March 2014

\begin{abstract}
We analyse geomagnetic recordings at four subauroral and midlatitude Russian observatories in $1850-1862$. The data consist of spot readings made once in hour of the north and east components of the magnetic field. We use the hourly change of the horizontal field vector as the measure of activity. We compare these values to data from modern observatories at corresponding magnetic latitudes (Nurmijärvi, Finland, magnetic latitude $\sim 57 \mathrm{~N}$; Tartu, Estonia, $\sim 54.5 \mathrm{~N}$; Dourbes, Belgium, $\sim 46 \mathrm{~N}$ ) by reducing their data to the 1-h format. The largest variations at the Russian observatories occurred during the Carrington storm in September 1859 and they reached about $1000 \mathrm{nT} / \mathrm{h}$, which was the instrumental off-scale limit. When the time stamp for the spot readings happens to be optimal, the top variation in the Nurmijärvi data is about $3700 \mathrm{nT} / \mathrm{h}$ (July 1982), and at Tartu the maximum is about $1600 \mathrm{nT} / \mathrm{h}$ (November 2004). At a midlatitude site Nertchinsk in Russia (magnetic latitude $\sim 45 \mathrm{~N}$ ), the variation during the Carrington storm was at the off-scale limit, and exceeded the value observed at Dourbes during the Halloween storm in October 2003. At Nertchinsk, the Carrington event was at least four times larger than any other storm in 1850-1862. Despite the limitations of the old recordings and in using only hourly spot readings, the Carrington storm was definitely a very large event at midlatitudes. At higher latitudes, it remains somewhat unclear whether it exceeds the largest modern storms, especially the one in July 1982.
\end{abstract}

Key words. extreme events - geomagnetism - historical records

\section{Introduction}

The Carrington storm in the end of August and beginning of September 1859 belongs to the largest space weather events in the known record (e.g., Tsurutani et al. 2003; Cliver \& Svalgaard 2004; Clauer \& Siscoe 2006; Cliver \& Dietrich 2013). If a similar storm happened now, it could cause wide damage to the modern technology in space and on the ground. Consequently, it is important to try to quantify the magnitude of the Carrington event based on fairly sparse historic data.

At those times, there were only a few observatories around the world that were capable to measure geomagnetic variations. The best data are obviously available from Colaba, India, showing a negative deflection up to about $-1600 \mathrm{nT}$ in the horizontal field (Tsurutani et al. 2003). When estimated in terms of the standard 1-h $D_{\text {st }}$ index ( $-850 \mathrm{nT}$; Siscoe et al. 2006), this event is definitely, but not extremely much larger than the next biggest one in March 1989 with $D_{\text {st }}$ of -589 nT. However, $D_{\text {st }}$ is only one measure of activity, and mostly related to the magnetospheric ring current. Besides, its determination from observations at a single site is somewhat controversial (e.g., Akasofu \& Kamide 2005).

In this paper, we consider variations of the ground magnetic field measured at midlatitude and subauroral observatories in Russia in 1850-1862, containing also fairly good data of the 1859 storm. We compare their magnitudes to those by modern instruments by reducing new data to the old format.

\section{Methods}

Around the mid of 1800 s, seven Russian observatories were in operation in the northern hemisphere (Nevanlinna \& Häkkinen 2010). We use four of them in this study covering the years 1850-1862 (Fig. 1, Table 1). The technical description of the instruments is given by Nevanlinna (1997). The data set consists of one value per hour for the magnetic north and east components $(H, D)$. The hourly value is the average of five visual observations at $15 \mathrm{~s}$ intervals centred at the particular time. This corresponds quite accurately to the modern 1-min values except that we only have one per hour instead of 60 per hour. When converted from the local time to UT, the time stamp is different from the full hour. Additionally, the reading time changed in the beginning of 1856 at all observatories. However, we considered the time series as a whole without separating it into two parts. Concerning the original time stamping, in the Russian yearbooks the time in hourly observations was given in the Göttingen time until 1856 (1857 in St. Petersburg) and later in local solar time (Nevanlinna \& Häkkinen 2010). Göttingen time leads by $0.66 \mathrm{~h}$ the present day Universal Time (UT).

The coverage of the data is very good throughout the period of 1850-1862. Especially, during the Carrington storm from 28 August to 3 September 1859, there are only a few missing values. Variations exceeding about $1000 \mathrm{nT}$ could not be detected by the instruments. As it will be seen, such off-scale values evidently occurred during the Carrington storm. 
Table 1. Coordinates of the observatories used in this study. Corrected geomagnetic coordinates (CGM) are given for the years 2000 and $1900^{\mathrm{a}}$. The last column gives the linearly extrapolated value for 1850 . For Nurmijärvi, Tartu and Dourbes, the CGM coordinates in 1850 and 1900 are irrelevant.

\begin{tabular}{llccccc}
\hline \hline Code & Full name & lat & lon & lat $_{\mathrm{m} 2000}$ & lat $_{\mathrm{m} 1900}$ & lat $_{\mathrm{m} 1850}$ \\
\hline STP & St. Petersburg & 59.93 & 30.30 & 56.16 & 54.73 & 54.01 \\
EKA & Ekaterinburg & 56.82 & 60.58 & 52.72 & 50.39 & 49.23 \\
BAR & Barnaul & 53.33 & 83.95 & 48.97 & 47.22 & 46.35 \\
NER & Nertchinsk & 51.32 & 119.60 & 45.87 & 45.30 & 45.02 \\
NUR & Nurmijärvi & 60.50 & 24.65 & 56.90 & - & - \\
TAR & Tartu & 58.26 & 26.46 & 54.48 & - & - \\
DOU & Dourbes & 50.1 & 4.6 & 45.88 & - \\
\hline
\end{tabular}

${ }^{a}$ http://omniweb.gsfc.nasa.gov/cgi/vitmo/vitmo_model.cgi

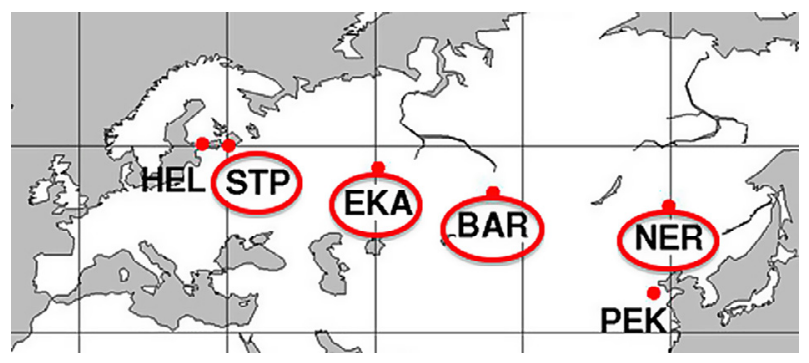

Fig. 1. Russian observatories in the 1800 s used in this study are marked by a red oval. Figure modified from Nevanlinna \& Häkkinen (2010).

The corrected geomagnetic coordinates (CGM) in Table 1 are important when comparing recordings at different locations at different times. Especially, the CGM latitudes in 1850-1862 were more southward than nowadays. A linear extrapolation back in time from the values of 1900 given in Table 1 shows that, for example, the CGM latitude at STP was about two degrees smaller in 1850 than in 2000. The present Tartu (TAR) observatory in Estonia has approximately an equal CGM latitude to that of STP around 1850s. As an additional reference, we used recordings from the Nurmijärvi (NUR) observatory, Finland. Digital data are available in 2002-2012 at TAR and in 1975-2012 at NUR. For comparisons with NER, we used data from the Dourbes observatory (DOU), Belgium, in 2002-2012. Its CGM latitude in 2000 is very close to that of NER in 1900 .

We used the data available online ${ }^{1}$. However, compared to Nevanlinna \& Häkkinen (2010), we performed a slightly more accurate conversion of the original recordings to get the transverse magnetic north $(H)$ and east $(E)$ components of the horizontal field vector $(H)$. For the east component, Nevanlinna \& Häkkinen (2010) applied the approximation

$$
E \approx H_{0} \tan D
$$

where $D$ is the declination given in degrees and $H_{0}$ is a fixed value (15,800-21,000 nT depending on the station). A more precise formula is

$$
E=\left(H_{0}+H\right) \tan D,
$$

where $H$ is the measured deviation (in $\mathrm{nT}$ ) of the magnetic north component from its quiet time value. In practice, $|H|$ is

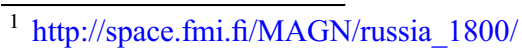

nearly always much smaller than $H_{0}$, so the approximation in equation (1) never differs significantly from the more accurate value given by equation (2).

\section{Results}

\subsection{Reduction of modern data to the 19th century format}

Modern recordings are typically available at least at a 1-min resolution. To make them comparable to the old Russian data, we reduced 1-min values to hourly spot readings. We constructed 60 new 1-h time series by taking every 60th value time-stamped at minutes $00,01, \ldots, 59$. A reasonable measure of magnetic activity is the time derivative of the horizontal field vector, calculated as the difference between two successive hourly values:

$$
|\mathrm{d} \mathbf{H} / \mathrm{d} t|=\sqrt{(\mathrm{d} X / \mathrm{d} t)^{2}+(\mathrm{d} Y / \mathrm{d} t)^{2}}
$$

Here $(X, Y)$ are the geographic north and east components of the magnetic field as is the modern standard. The vector derivative $|\mathrm{d} \mathbf{H} / \mathrm{d} t|$ is independent of the coordinate system and thus directly comparable to the corresponding quantity derived from the transverse horizontal components $(H, E)$ of the Russian recordings:

$$
|\mathrm{d} \mathbf{H} / \mathrm{d} t|=\sqrt{(\mathrm{d} H / \mathrm{d} t)^{2}+(\mathrm{d} E / \mathrm{d} t)^{2}} .
$$

Figure 2 shows examples of the reduced time series of $X$ at NUR on 12-15 July 1982, when the largest 1-min time derivatives were reached during the whole study period of 1975-2012. Figure 3 shows the maximum hourly $|\mathrm{d} \mathbf{H} / \mathrm{d} t|$ during the same event for different minutes used for the spot readings. Depending on the selected time step, there is a large range in the variations in the hourly values. For this specific event, it is very critical if the sharp peak on 13 July centred at 23:53 UT happens to be detected or not. We also made a simple test by applying an artificial off-scale limit to the data (Appendix A). This shows that such a limit can significantly decrease the values of $|\mathrm{d} \mathbf{H} / \mathrm{d} t|$.

Figure 4 shows the largest value of $|\mathrm{d} \mathbf{H} / \mathrm{d} t|$ for each 1-h time series for the full period of 1975-2012. The largest hourly change of about $3700 \mathrm{nT} / \mathrm{h}$ (minute 53, 13 July 1982) is a little more than twice the minimum one. We note that equally large variations in $X$ occurred during the same storm at more southern observatories in Lovö, Sweden (CGM lat $~ 56$ ), and in Brorfelde, Denmark (CGM lat 52) (Kappenman 2005, 2006). 
A. Viljanen et al.: Geomagnetic Variations in Russia in 1850-1862
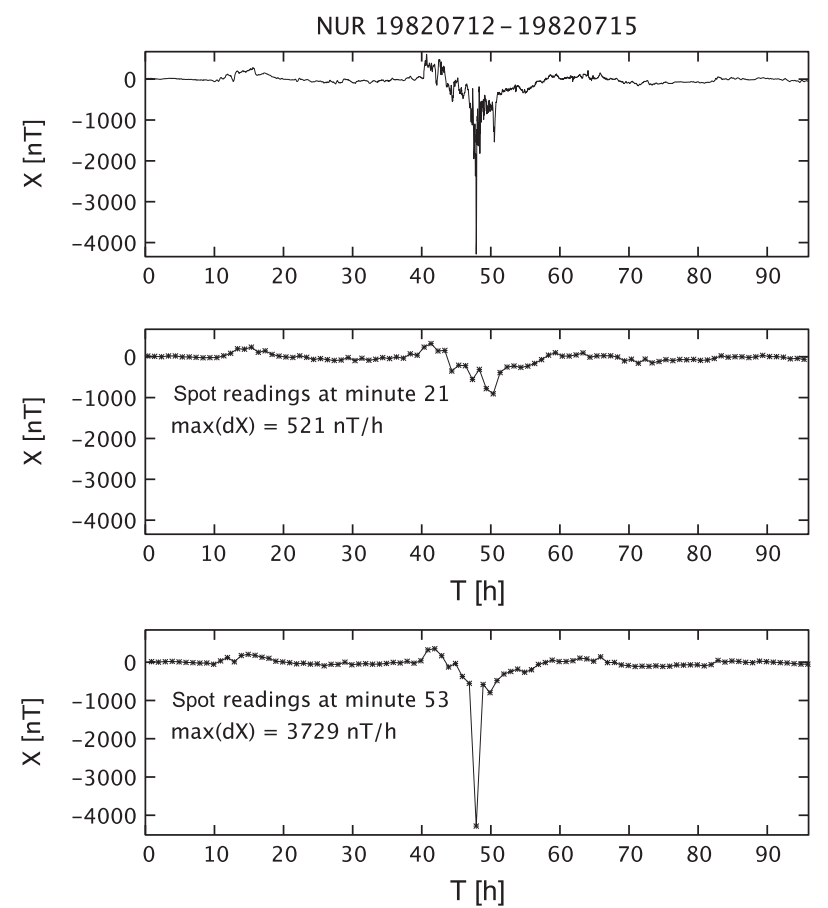

Fig. 2. Top: Original 1-min values of the $X$ component at NUR on 12-15 July 1982. The deepest peak occurs on 13 July at 23:53 UT. Centre: 1 -h time series by spot readings at minute 21 . Bottom: 1 -h time series by spot readings at minute 53 .

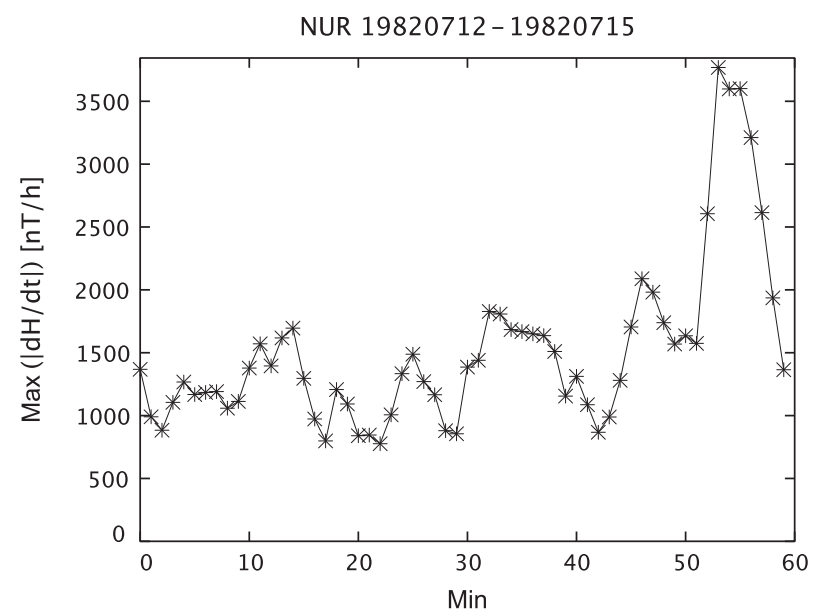

Fig. 3. Maximum hourly $|\mathrm{d} \mathbf{H} / \mathrm{d} t|$ at NUR on $12-15$ July 1982. The horizontal axis is the minute used for the hourly spot readings.

To check how well 1-h values can reveal the largest events defined by shorter time steps, we listed the 1-min maxima of $|\mathrm{d} \mathbf{H} / \mathrm{d} t|$ at NUR in Table 2. Then, for each of the sixty 1-h time series, we found the day on which $|\mathrm{d} \mathbf{H} / \mathrm{d} t|$ reached its maximum. The results are given in Table 3 . This gives eight different days as the most intense events. They also appear in the ranking list based on 1-min values in Table 2. However, the days (13 July 1982, 30 October 2003) with the largest 1-min daily maximum of $|\mathrm{d} \mathbf{H} / \mathrm{d} t|$ are not the same as the days with the largest occurrence of the maximum values of $1-\mathrm{h}|\mathrm{d} \mathbf{H} / \mathrm{d} t|$.

As already mentioned, the present magnetometre station at TAR corresponds to the old STP according to the geomagnetic latitudes at different times. The regular diurnal variation (Fig. 5)

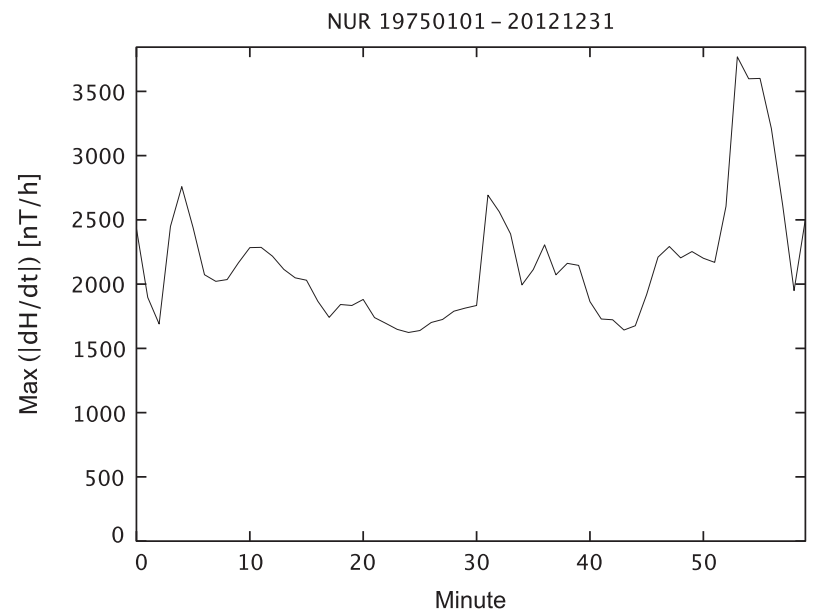

Fig. 4. Maximum hourly $|\mathrm{d} \mathbf{H} / \mathrm{d} t|$ at NUR in $1975-2012$ for 1-h times series corresponding to spot readings at minutes $00,01, \ldots, 59$ (horizontal axis).

Table 2. Top 20 days according to the maximum 1-min value of $|\mathrm{d} \mathbf{H} / \mathrm{d} t|$ at NUR in 1975-2012.

\begin{tabular}{lc}
\hline \hline yyyymmdd & Max $[\mathrm{nT} / \mathrm{min}]$ \\
\hline 19820713 & 1194.7 \\
20031030 & 1008.3 \\
19910324 & 941.8 \\
19860208 & 877.8 \\
19910325 & 800.0 \\
19820714 & 689.3 \\
19890313 & 647.5 \\
20031029 & 602.7 \\
20020907 & 596.7 \\
19891021 & 592.2 \\
19950407 & 579.4 \\
20041109 & 565.8 \\
19911028 & 557.9 \\
19911108 & 503.9 \\
20120315 & 493.1 \\
19911109 & 484.4 \\
20031014 & 464.0 \\
20021001 & 454.7 \\
20041108 & 435.9 \\
19890314 & 423.1 \\
\hline
\end{tabular}

Table 3. Days on which the 1-h time series of spot readings at minutes $00,01, \ldots, 59$ reach their maximum $|\mathrm{d} \mathbf{H} / \mathrm{d} t|$ at NUR in 1975-2012. The number of maxima on the particular day is also given.

\begin{tabular}{lr}
\hline \hline yyyymmdd & $\#$ \\
\hline 19860208 & 14 \\
20041109 & 14 \\
19890313 & 13 \\
20031030 & 6 \\
19820713 & 6 \\
20041108 & 4 \\
19910324 & 2 \\
19910325 & 1 \\
\hline
\end{tabular}

supports this, and also shows that the instruments at STP obviously produced reliable data. Since the equipment was identical at all Russian observatories, we can consider the data quality as good (Nevanlinna \& Häkkinen 2010). 

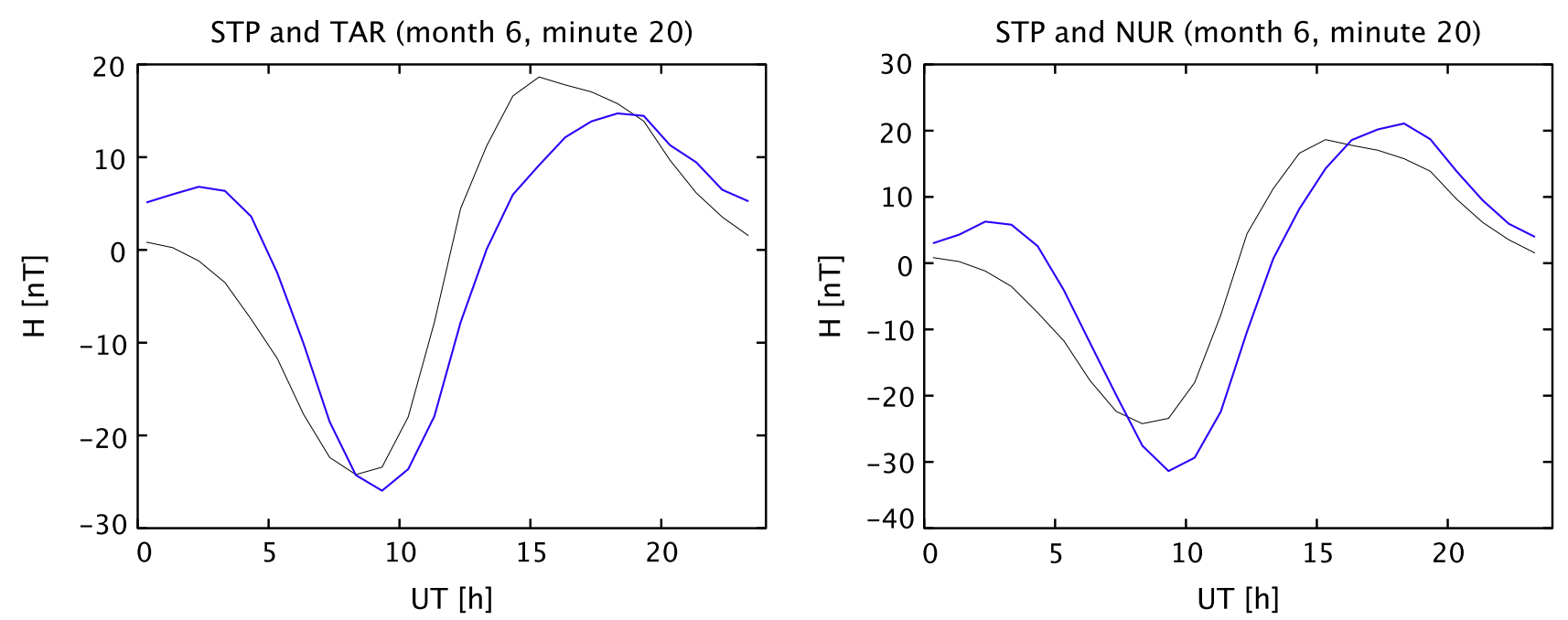

Fig. 5. Average diurnal variation of the magnetic north component $(H)$ at TAR and STP (left) and at NUR and STP (right). Black curves correspond to STP and blue curves to NUR and TAR. Results for the eastward component $(E)$, not plotted here, show an equally good similarity. We assumed that the declination at NUR and TAR is $5.5^{\circ}$ (positive eastwards) when determining the magnetic north and east components from the geographic components $(X, Y)$.

For TAR and DOU, we constructed time series of 1-h spot readings in 2002-2012 in the same way as for NUR. The maximum $|\mathrm{d} \mathbf{H} / \mathrm{d} t|$ for different minute values is shown in Figure 6, with results for NUR of the same period of 2002-2012 for comparison. The largest hourly change at TAR (minute 20) is a little more than twice the minimum one. The corresponding feature was found above for NUR in 1975-2012, and it is also visible at NUR for the shorter interval 2002-2012. It is noteworthy that the smallest value at NUR is still nearly equal to the largest one at TAR. There seems to be quite a rapid decrease in geomagnetic activity toward lower geomagnetic latitudes at around the magnetic latitude of $55 \mathrm{~N}$. A similar finding was noted by Thomson et al. (2011) and Pulkkinen et al. (2012).

Concerning DOU, the maximum $|\mathrm{d} \mathbf{H} / \mathrm{d} t|$ of about $900 \mathrm{nT} / \mathrm{h}$ is reached if the spot readings are made at the minute 59 . This value is only slightly larger than the smallest one at TAR. Again, the selection of the time stamp for hourly readings affects the maximum $|\mathrm{d} \mathbf{H} / \mathrm{d} t|$ by a factor of two. We also note that all values at DOU shown in Figure 6, occur on 29-30 October 2003, and most of them at the beginning of the storm at around 06 UT on 29 October.

\subsection{Largest events in the Russian data in 1850-1862}

Figure 7 shows the recordings at STP and NER during the Carrington storm from 28 August to 3 September 1859 (see also Nevanlinna 2008; Tyasto et al. 2009; Ptitsyna et al. $2012 \mathrm{a}, \mathrm{b})$. There are prominent variations on 2 September 1859 which are close to the off-scale threshold. It is very likely that the observer could not perfectly follow visually the rapid large oscillations of the magnets, and the true variations probably exceeded $1000 \mathrm{nT}$. Concerning STP, there are four completely missing values around the midnight on 28-29 Aug and one missing value on 3 September. The largest variations available on 2 September seem to be clearly below the off-scale limit.

We point out again that the values of the $1-h$ variation depend on which minute happens to be selected for the spot readings. Based on the results for the modern observatories, we can speculate that the largest hourly changes at the Russian sites could have been twice the values shown here, added by an unknown amplitude concerning the off-scale cases.

Figure 8 shows the daily maximum of 1-h changes of $H$ at the Russian observatories in the whole study period. The Carrington storm in September 1859 is the largest peak at all sites. However, there are nearly equal peaks at EKA at other days. Only at NER, the Carrington event is clearly dominating. At BAR and EKA, the hourly change reached 400-500 nT during the Carrington storm, which is not strikingly large compared to other events at these sites. However, there are a few missing values at BAR and EKA on 2 September 1859 , so the maximum phases of the storm might have been lost at these stations.

STP is of a special interest when compared to the recordings at NUR and TAR. Especially, the geomagnetic latitude of TAR is very close to that at STP in 1850. Figure 9 shows $|\mathrm{d} \mathbf{H} / \mathrm{d} t|$ at TAR in 2002-2012 for spot readings made at minutes 20 and 41 . The former corresponds to the largest value of maximum $|\mathrm{d} \mathbf{H} / \mathrm{d} t|$ as seen in Figure 6, and the latter corresponds to the smallest value, respectively. At STP in 1850-1862, the second largest $|\mathrm{dH} / \mathrm{d} t|$ was about $700 \mathrm{nT} / \mathrm{h}$ in 1862 . The corresponding maximum at TAR in 2002-2012 is larger than this for any minute selected for the hourly time stamp, being mostly larger than $1000 \mathrm{nT} / \mathrm{h}$ (Fig. 6). Only the Carrington storm at STP reached an equal magnitude. This shows that in terms of maximum $|\mathrm{d} \mathbf{H} / \mathrm{d} t|, 2002-2012$ was in general magnetically more active than 1850-1862, with the Carrington storm possibly being an exception.

We remark that the Halloween storm in October 2003 was not the largest event at TAR, but a bigger one occurred there on 9 November 2004 (nearly $1600 \mathrm{nT} / \mathrm{h}$ for spot readings at minute 20). However, when considering 1-min values, the Halloween storm still has the largest $|\mathrm{d} \mathbf{H} / \mathrm{d} t|$ on 29 October 2003. This also shows that the reduction of 1-min data to 1-hour spot readings causes some bias.

At DOU (Fig. 10), the Halloween storm was clearly the largest event there in 2002-2012. However, it is not as pronounced as the Carrington storm at NER (Fig. 8) when com- 

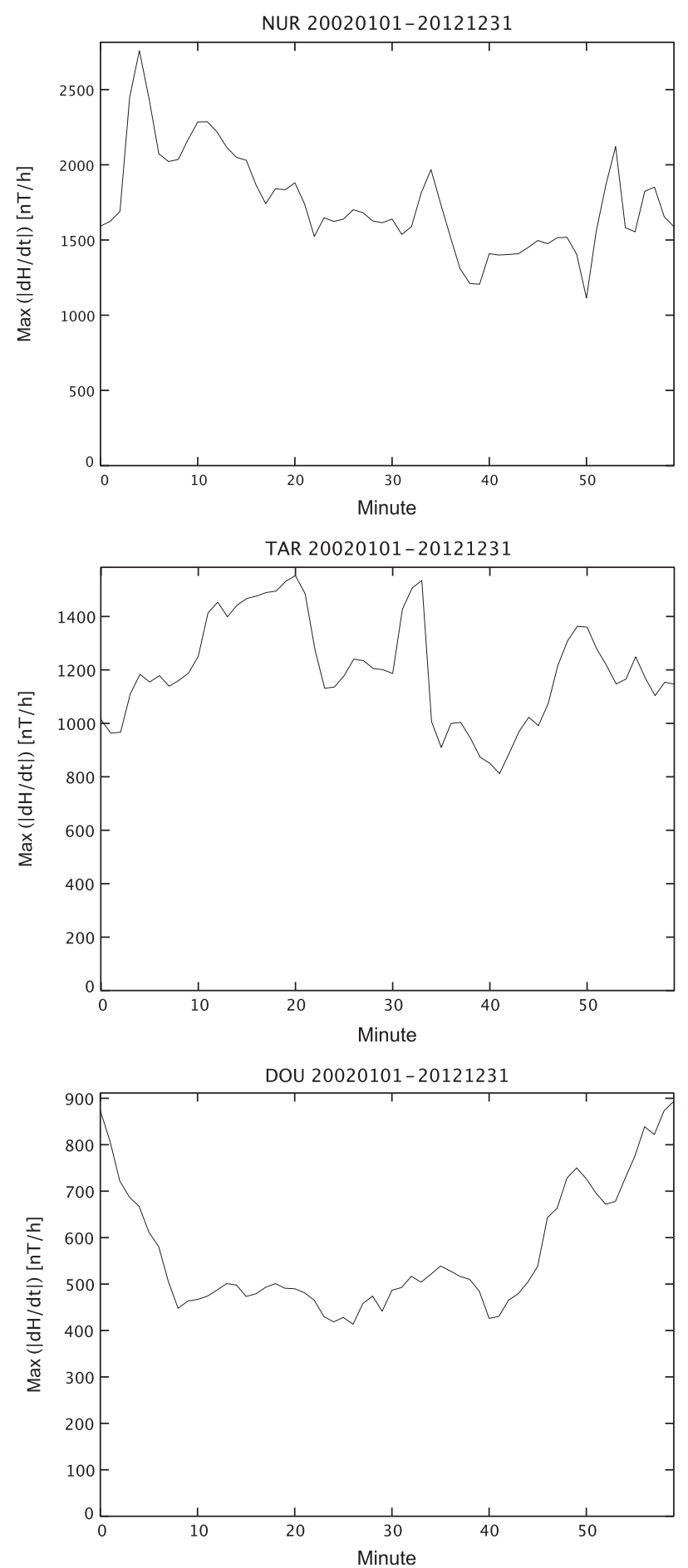

Fig. 6. From top to bottom: Maximum hourly $|\mathrm{d} \mathbf{H} / \mathrm{d} t|$ at NUR, TAR and DOU in 2002-2012 for 1-h times series starting at minutes 00, $01, \ldots, 59$ (horizontal axis). Compare also to Figure 4.

pared to other events during the period under study. It remains unclear to what extent the true variation at NER exceeded $1000 \mathrm{nT} / \mathrm{h}$. All hourly readings of the storm are available with the largest value being $1044 \mathrm{nT}$ for the magnetic east component, and simultaneously $115 \mathrm{nT}$ for the north component, as calculated from equation (2). We cannot know whether the observer noted an off-scale event and saved the largest value shown in the instrument scale or whether the variation happened to be just very close to the off-scale limit. In any case, $|\mathrm{d} \mathbf{H} / \mathrm{d} t|$ at NER was larger than the top value of about $900 \mathrm{nT} / \mathrm{h}$ at DOU during the Halloween storm.
The most prominent difference between STP and NUR is that the latter has clearly larger $|\mathrm{d} \mathbf{H} / \mathrm{d} t|$ being at least about $1600 \mathrm{nT} / \mathrm{h}$ and reaching even about $3750 \mathrm{nT} / \mathrm{h}$ depending on which minute is used for the 1-h time series (Fig. 4). At STP, the top value is about $1000 \mathrm{nT} / \mathrm{h}$, which is only about $30 \%$ of the maximum at NUR in 1975-2012. The difference between STP and NUR remains large even if we consider only data of the 11-year period of 2002-2012 at NUR in Figure 6. It is noteworthy that NER reaches an equally large value at about $10^{\circ}$ more southward in geomagnetic latitudes. The Carrington storm was there about four times larger than any other event in 1851-1862 as measured by the hourly change in the horizontal field vector.

As a comparison, we note the large storm observed at Greenwich on 24 October 1847 (Fig. 8 in Cliver \& Dietrich 2013). There were no off-scale problems, and the range of the horizontal field was about $1500 \mathrm{nT}$, which exceeds the values available from the Russian stations in 1850-1862. The geomagnetic latitude of Greenwich (in 1900) was roughly $50^{\circ}$, so it corresponds to a station between the present Dourbes and Tartu. Comparison to Figure 9 indicates that the 1847 storm was equal to or even larger than the October 2003 and November 2004 storms at the geomagnetically more northern Tartu. In 1882 to 1938 , there were a few other storms observed at Greenwich and Potsdam (Germany) with equal magnitudes (up to $1900 \mathrm{nT}$ ) to the Greenwich 1847 event (Table 1 in Tsurutani et al. 2003). All these storms are still much smaller than the July 1982 event at Nurmijärvi, Lovö and Brorfelde.

\section{Conclusions}

Reduction of modern 1-min recordings to 1-h spot readings gives a quantitative reference to the hourly spot readings at Russian observatories in 1850-1862. Results based on the modern data show that the maximum $|\mathrm{d} \mathbf{H} / \mathrm{d} t|$ varies somewhat randomly depending on which minute is selected as the time stamp for the hourly readings. It is possible that the maximum might have been about two times larger than the value available from the historic records.

Comparison of the recordings at St. Petersburg (STP) to the nearby present observatories at Nurmijärvi (NUR) and Tartu (TAR) shows that the Carrington storm in 1859 was not necessarily extraordinarily large there. Especially, NUR has clearly larger values of $|\mathrm{d} \mathbf{H} / \mathrm{d} t|$ than STP and TAR independent of the selected time stamp for hourly readings. However, there were obviously off-scale values at STP during the most intense phase of the event, thus leaving some vagueness to this conclusion.

On the contrary, at Nertchinsk (NER) located at about $10^{\circ}$ more southward in geomagnetic latitudes, the Carrington event was at least at the same level as the Halloween storm in October 2003 at TAR. Additionally, the top value of $|\mathrm{d} \mathbf{H} / \mathrm{d} t|$ at NER was at least four times larger than during any other event there in 1851-1862. However, we note that this result could be different if another minute had been used for the spot readings. When compared to Dourbes (DOU) located at about the same magnetic latitude as NER, the Carrington storm was larger than the Halloween storm. Consequently, it is quite obvious that the Carrington storm was an exceptionally huge event at geomagnetic mid-latitudes $\left(\sim 45^{\circ} \mathrm{N}\right)$. Still, it might have been smaller there than the largest storms at NUR, for example. 

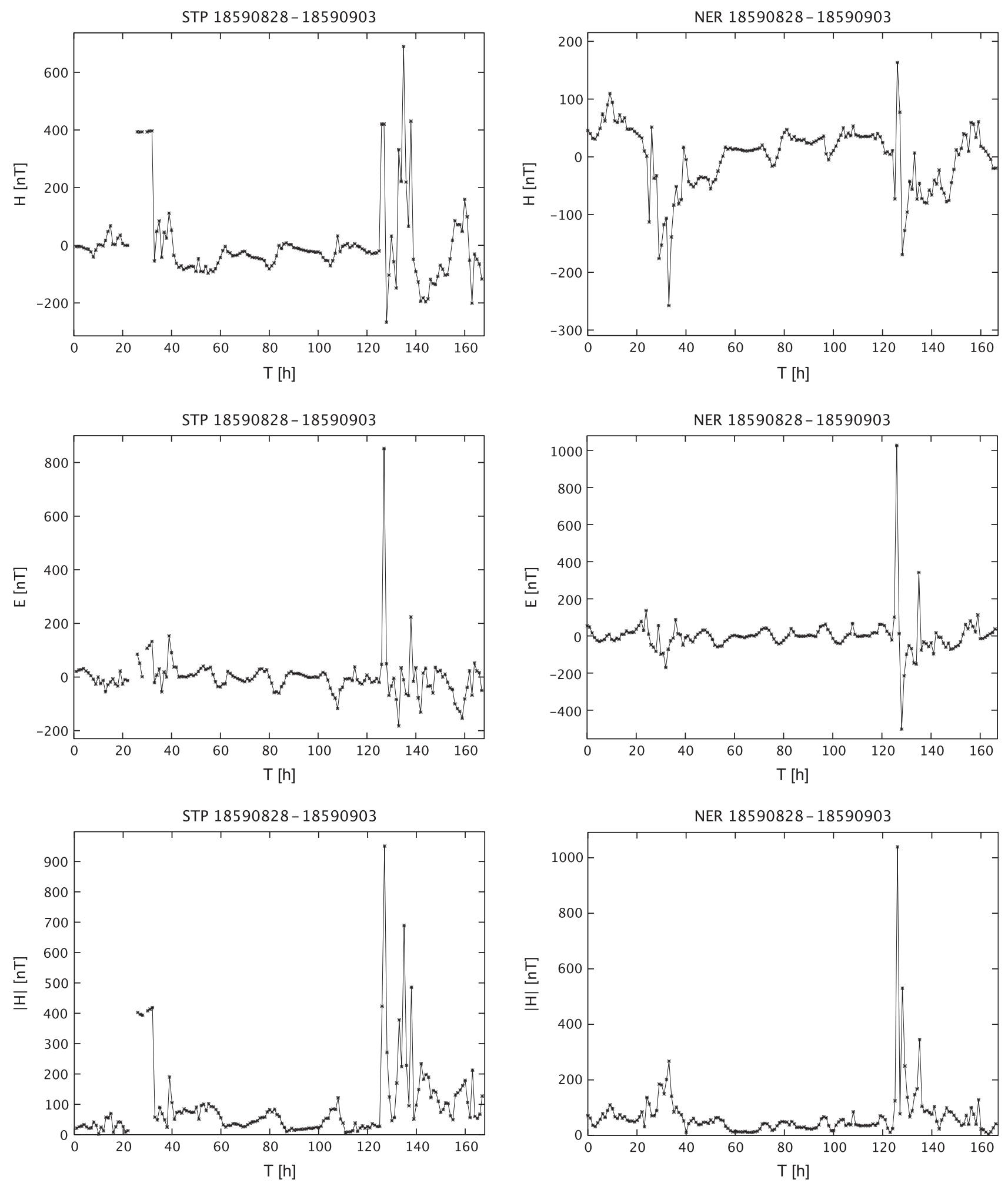

Fig. 7. Recordings of $H, E$ and $|\mathbf{H}|=\sqrt{H^{2}+E^{2}}$ in 28 August to 3 September 1859. Left: STP, right: NER.

We can even speculate that the Carrington event is not the largest one observed at (sub)auroral latitudes. An alternative candidate is the storm on 13-14 July 1982. There are also suggestions that the May 1921 storm might have been a very extreme one (Kappenman 2006; Cliver \& Dietrich 2013). On the other hand, Loomis (1860) reported that a variation of the horizontal field up to about one-eighth of its mean value occurred on 2 September 1859 at Rome. This is about
3000 nT (Boteler 2006; Ptitsyna \& Altamore 2012; Ptitsyna et al. 2012a), which would be an exceptionally large value even at higher latitudes (cf. variations at NUR in Fig. 2).

Some original $15 \mathrm{~s}$ readings during the Carrington storm are also available from the Helsinki observatory (HEL in Fig. 1). Before the instruments went off-scale, time derivatives of about $420 \mathrm{nT} / 15 \mathrm{~s}$ in $D$ and $250 \mathrm{nT} / 15 \mathrm{~s}$ in $H$ were reached (Nevanlinna 2008). The $D$ variation is equal to the maximum 
A. Viljanen et al.: Geomagnetic Variations in Russia in 1850-1862
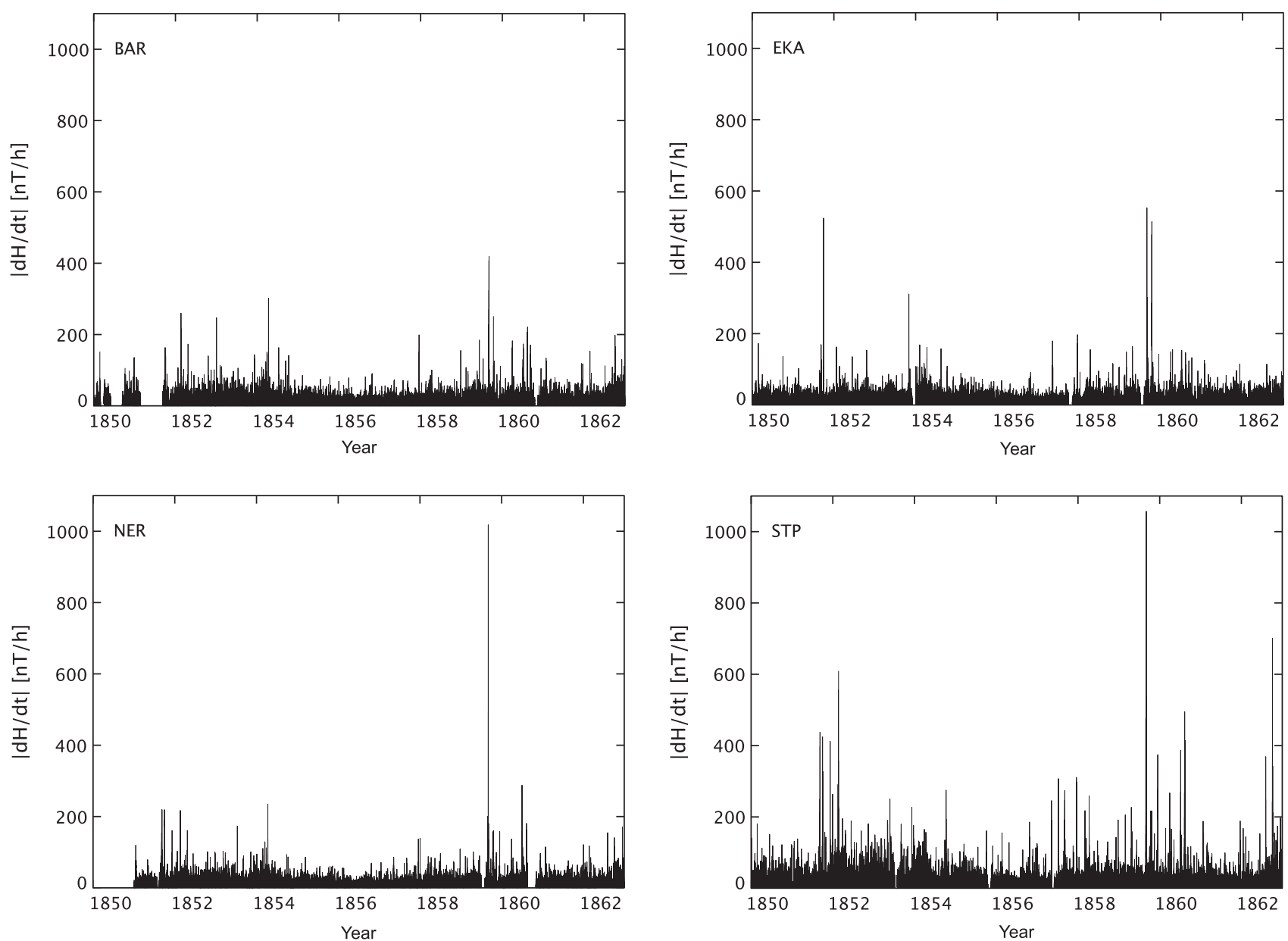

Fig. 8. All values of $|\mathrm{d} \mathbf{H} / \mathrm{d} t|$ at BAR, EKA, NER and STP in $1850-1862$ for 1 -h spot readings.
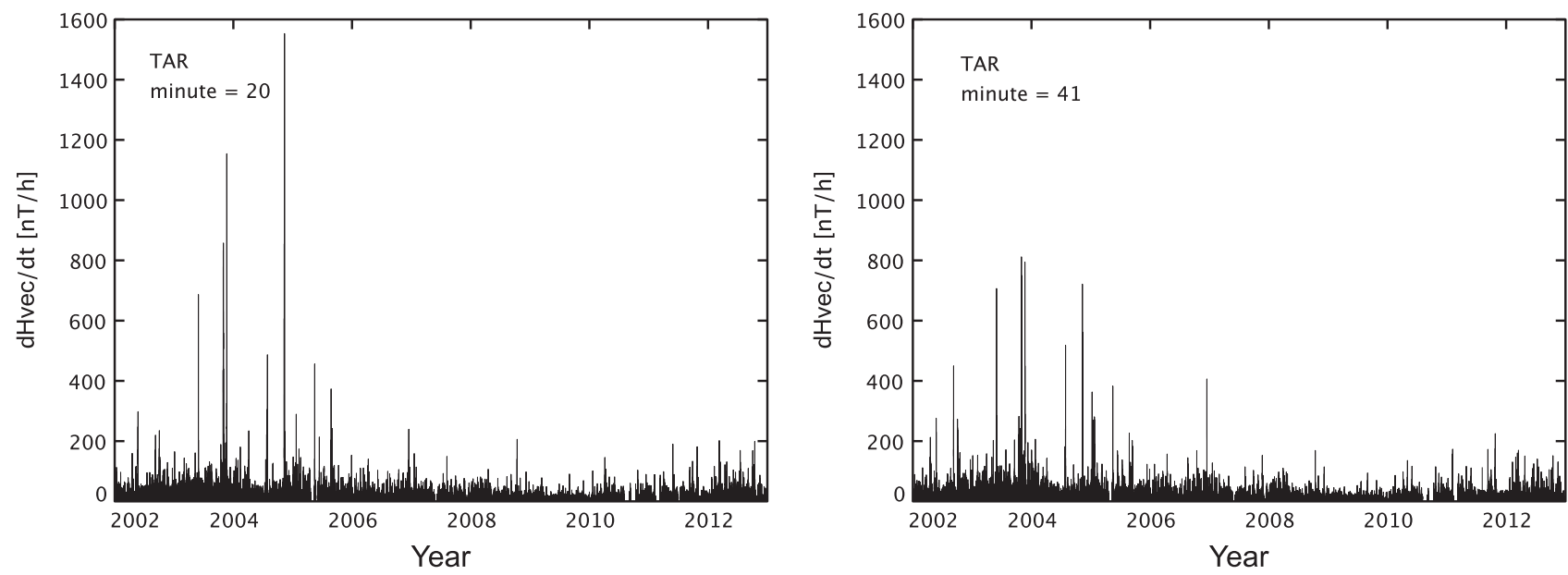

Fig. 9. All values of $|\mathrm{d} \mathbf{H} / \mathrm{d} t|$ at TAR in 2002-2012 for 1-h spot readings at minutes 20 (left) and 41 (right).

value of $28 \mathrm{nT} / \mathrm{s}$ in $|\mathrm{d} \mathbf{H} / \mathrm{d} t|$ (10-s value) at the Nurmijärvi observatory, close to Helsinki, during the Halloween storm. However, the largest known $|\mathrm{d} \mathbf{H} / \mathrm{d} t|$ at Nurmijärvi is $40 \mathrm{nT} / \mathrm{s}$ on 13-14 July 1982 (Viljanen 1997). Again, it remains unresolved whether the values from HEL represent the true maxima during the Carrington storm or whether larger ones occurred during the off-scale periods.
Acknowledgements. The research leading to these results has received funding from the European Community's Seventh Framework Programme (FP7/2007-2013) under grant agreement No. 260330. Data of the Dourbes observatory were provided by Centre de Physique du Globe, Institute Royal Meteorologique, Belgium, and downloaded from the World Data Centre for Geomagnetism (Edinburgh). We thank Dr. Risto Pirjola and the two referees for comments clarifying this paper. 

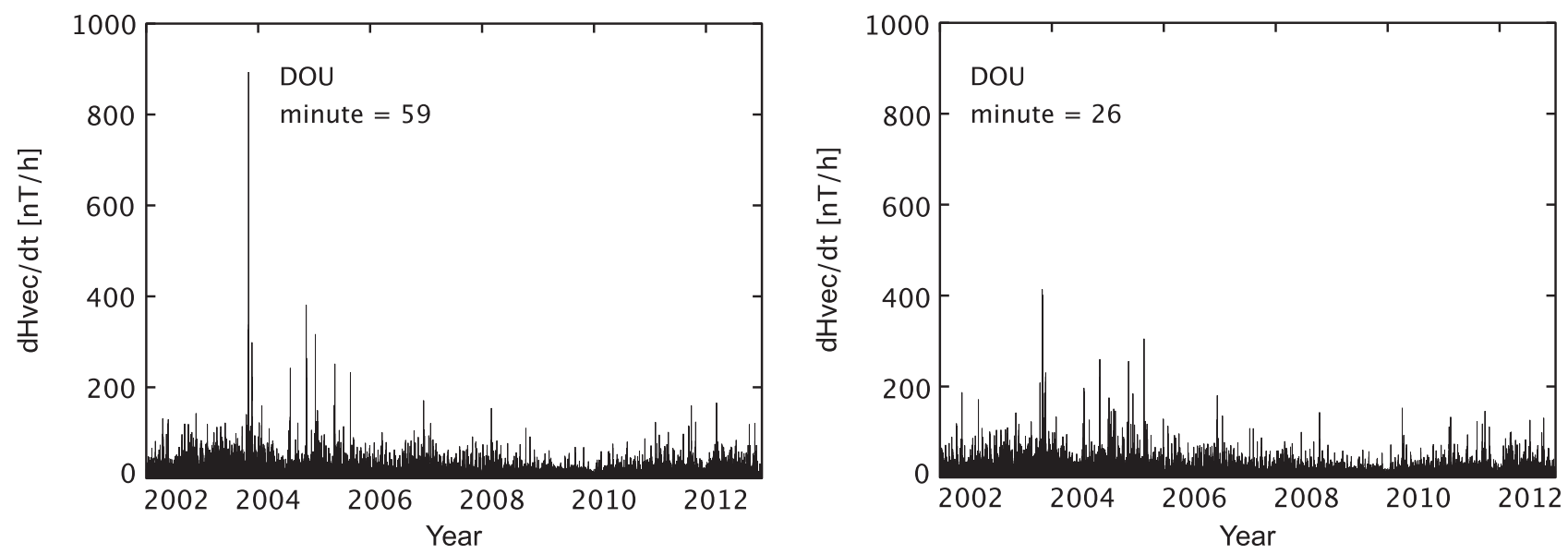

Fig. 10. All values of $|\mathrm{d} \mathbf{H} / \mathrm{d} t|$ at DOU in 2002-2012 for 1-h spot readings at minutes 59 (left) and 26 (right).
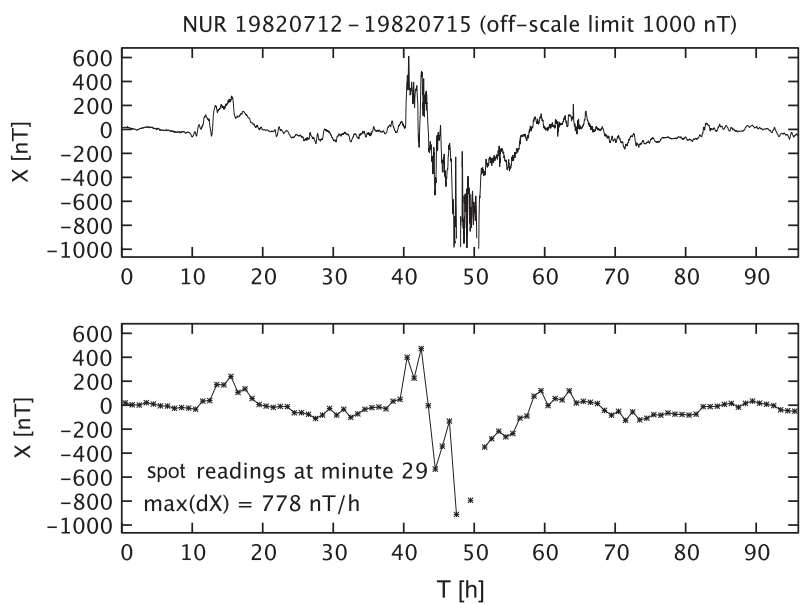

Fig. 11. Top: 1-min values of $X$ at NUR on $12-15$ July 1982, but all variations exceeding $1000 \mathrm{nT}$ assumed to be missing. Bottom: 1-h time series by spot readings at minute 29 , which now gives the largest $\mathrm{d} X / \mathrm{d} t$ (cf. Fig. 2).

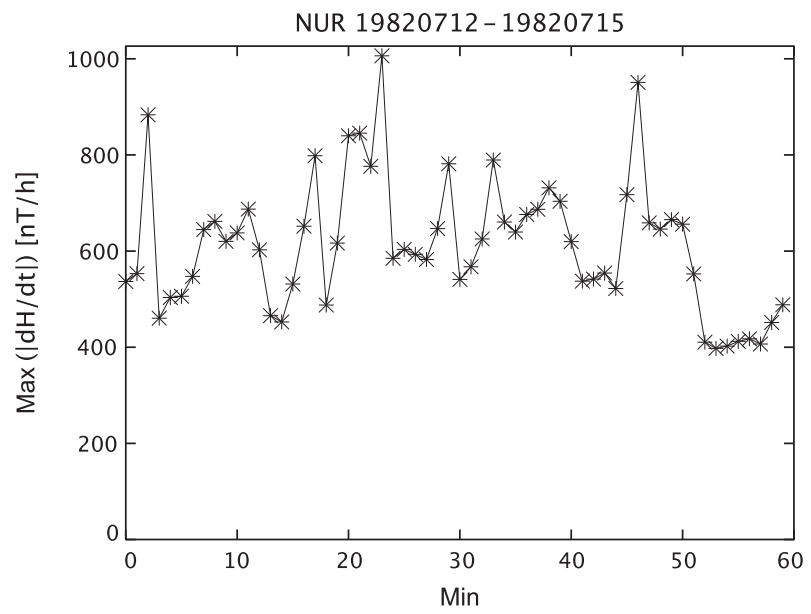

Fig. 12. Maximum hourly $|\mathrm{d} \mathbf{H} / \mathrm{d} t|$ at NUR on $12-15$ July 1982 when assuming an off-scale limit as in Figure 11. The horizontal axis is the minute used for the hourly spot readings (cf. Fig. 3).

\section{References}

Akasofu, S.-I., and Y. Kamide, Comment on "The extreme magnetic storm of 1D2 September 1859" by B.T. Tsurutani, W.D. Gonzalez, G.S. Lakhina, and S. Alex, J. Geophys. Res., 110, A09226, DOI: 10.1029/2005JA011005, 2005.

Boteler, D.H., The super storms of August/September 1859 and their effects on the telegraph system, Adv. Space Res., 38, 159-172, DOI: 10.1016/j.asr.2006.01.013, 2006.

Clauer, C.R., and G. Siscoe (Eds.), The great historical geomagnetic storm of 1859: a modern look, Adv. Space Res., 38, DOI: 10.1016/j.asr.2006.09.001, 2006.

Cliver, E.W., and W.F. Dietrich, The 1859 space weather event revisited: limits of extreme activity, J. Space Weather Space Clim., 3, A31, DOI: 10.1051/swsc/2013053, 2013.

Cliver, E.W., and L. Svalgaard, The 1859 solar-terrestrial disturbance and the current limits of extreme space weather activity, Sol. Phys., 224, 407-422, DOI: 10.1007/s11207-005-4980-z, 2004.

Kappenman, J.G., An overview of the impulsive geomagnetic field disturbances and power grid impacts associated with the violent Sun-Earth connection events of 29-31 October 2003 and a comparative evaluation with other contemporary storms, Space Weather, 3, S08C01, DOI: 10.1029/2004SW000128, 2005.

Kappenman, J.G., Great geomagnetic storms and extreme impulsive geomagnetic field disturbance events - an analysis of observational evidence including the great storm of May 1921, $A d v$. Space Res., 38, 188-199, DOI: 10.1016/j.asr.2005.08.055, 2006.

Loomis, E., The great auroral exhibition of August 28th to September 4th, 1859, Am. J. Sci. Arts, 4th article: XXIX, 386399, 1860.

Nevanlinna, H., Gauss' H-Variometer at the Helsinki Magnetic Observatory (1844-1912), J. Geomagn. Geoelectr., 49, 12091216, DOI: 10.5636/jgg.49.1209, 1997.

Nevanlinna, H., On geomagnetic variations during the AugustSeptember storms of 1859, Adv. Space Res., 42, 171-180, DOI: 10.1016/j.asr.2008.01.002, 2008.

Nevanlinna, H., and L. Häkkinen, Results of Russian geomagnetic observatories in the 19th century: magnetic activity, 1841-1862, Ann. Geophys., 28, 917-926, DOI: 10.5194/angeo-28-917-2010, 2010.

Ptitsyna, N., and A. Altamore, Father Secchi and the first Italian magnetic observatory, Hist. Geo Space. Sci., 3, 33-45, DOI: 10.5194/hgss-3-33-2012, 2012.

Ptitsyna, N.G., M.I. Tyasto, and A. Altamore, New data on the giant September 1859 magnetic storm: an analysis of Italian and Russian historic observations, in: Proceedings of the 9th Intl. Conf. "Problems of Geocosmos", Oct 8-12, 2012, St. Petersburg, Russia, pp. 353-358, 2012a. 
A. Viljanen et al.: Geomagnetic Variations in Russia in 1850-1862

Ptitsyna, N.G., M.I. Tyasto, and B.A. Khrapov, Great geomagnetic storms in 1841-1870 according to the data from the network of russian geomagnetic observatories, Geomagn. Aeron., 52, 649659, DOI: 10.1134/S0016793212040123, 2012b.

Pulkkinen, A., E. Bernabeu, J. Eichner, C. Beggan, and A.W.P. Thomson, Generation of 100-year geomagnetically induced current scenarios, Space Weather, 10, S04003, DOI: 10.1029/2011SW000750, 2012.

Siscoe, G., N.U. Crooker, and C.R. Clauer, $D_{\mathrm{st}}$ of the Carrington storm of 1859, Adv. Space Res., 38, 173-179, DOI: 10.1016/j.asr.2005.02.102, 2006.

Thomson, A., E. Dawson, and S. Reay, Quantifying extreme behaviour in geomagnetic activity, Space Weather, 9, S10001, DOI: 10.1029/2011SW000696, 2011.

Tsurutani, B.T., W.D. Gonzalez, G.S. Lakhina, and S. Alex, The extreme magnetic storm of 1-2 September 1859, J. Geophys. Res., 108, 1268, DOI: 10.1029/2002JA009504, 2003.

Tyasto, M.I., N.G. Ptitsyna, I.S. Veselovsky, and O.S. Yakovchouk, Extremely strong geomagnetic storm of September 2-3, 1859, According to the archived data of observations at the Russian network, Geomagn. Aeron., 49, 153-162, DOI: $10.1134 / \mathrm{S} 0016793209020030,2009$

Viljanen, A., The relation between geomagnetic variations and their time derivatives and implications for estimation of induction risks, Geophys. Res. Lett., 24, 631-634,

DOI: 10.1029/97GL00538, 1997.

\section{Appendix A: Off-scale test with modern magnetometre data}

We simulated the effect of the instrumental off-scale limit on modern recordings as follows. We considered 1-min time series and subtracted a quiet-time baseline. Whenever the remaining variation field values exceeded $1000 \mathrm{nT}$, we marked them as missing values. As an example, we considered again the extreme storm in July 1982 at NUR. The artificial cutting of large variations changes the $X$ magnetogram quite radically (Fig. 11) when compared to the true observations (Fig. 2). As is natural, $|\mathrm{d} \mathbf{H} / \mathrm{d} t|$ also decreases much as shown in Figure 12. Theoretically, the largest $|\mathrm{d} \mathbf{H} / \mathrm{d} t|$, within the assumed off-scale limit, is reached when the successive $\mathbf{H}$ vectors are antiparallel and $|X|=|Y|=1000 \mathrm{nT}$. Then the maximum $|\mathrm{d} \mathbf{H} / \mathrm{d} t|$ is $2 \cdot \sqrt{2} \cdot 1000 \mathrm{nT} / \mathrm{h} \approx 2800 \mathrm{nT} / \mathrm{h}$

This simple test shows that if the Carrington storm was comparable to the July 1982 event then the instrumental off-scale limit caused a significant gap in large $|\mathrm{d} \mathbf{H} / \mathrm{d} t|$ values. However, it will evidently remain completely unknown how large the true variations were at the Russian stations.

Cite this article as: Viljanen A, Myllys M \& Nevanlinna H: Russian geomagnetic recordings in 1850-1862 compared to modern observations. J. Space Weather Space Clim., 2014, 4, A11. 